\title{
États limites et bouffées turbulentes en conduite cylindrique
}

\author{
Yohnn Duguet ${ }^{1,3, a}$, A.P. Willis ${ }^{2,3}$ et R.R. Kerswell ${ }^{3}$ \\ 1 Laboratoire d'Informatique pour la Mécanique et les Sciences de l'Ingénieur (LIMSI-CNRS), UPR 3251, BP 133, \\ 91403 Orsay Cedex, France \\ 2 LadHyX, École Polytechnique, 91128 Palaiseau, France \\ 3 School of Mathematics, University of Bristol BS8 1TW, Bristol, UK
}

Reçu le 17 juillet 2009, accepté le 22 février 2010

\begin{abstract}
Résumé - La transition vers la turbulence en conduite cylindrique est observée pour Re assez grand, malgre la stabilité linéaire de l'état laminaire. Expérimentalement, la transition se manifeste par le développement de bouffées turbulentes, spatialement localisées. Du côté théorique, des ondes progressives, instables et de courte longueur d'onde, ont été mises en évidence numériquement. Cette étude, qui utilise la simulation numérique directe ainsi qu'un modèle réduit, suggère la compatibilité entre les deux approches.
\end{abstract}

Mots clés : Transition / turbulence / conduite cylindrique / frontière laminaire-turbulent / état limite

\begin{abstract}
Edge states and slugs in cylindrical pipe flow. Transition to turbulence in cylindrical pipe flow is observed for large enough values of $R e$, despite the linear stability of the laminar base flow. Experimentally, transition occurs through the growth of localised structures like puffs or slugs. On a more theoretical side, unstable travelling waves with a short wavelength have been computed numerically. This investigation, based on both between direct numerical simulation and a reduced order model, suggests compatibility between the two approaches.
\end{abstract}

Key words: Transition / turbulence / pipe flow / laminar-turbulent boundary / edge state

\section{Introduction}

\subsection{Problématique}

Le problème étudié ici est celui de la transition vers la turbulence dans un écoulement de fluide incompressible à l'intérieur d'une conduite cylindrique. L'écoulement peut être généré par un gradient de pression imposé, ou forcé par un débit constant (par exemple via l'action d'un piston, comme dans une seringue). Le cas considéré ici est celui d'un débit constant [1]. Ce problème est caractérisé par le nombre de Reynolds $R e=\frac{U D}{\nu}$, où $U, D$ et $\nu$ sont respectivement la vitesse de débit, le diamètre de la conduite et la viscosité cinématique du fluide. Pour des valeurs faibles de Re, l'écoulement est stationnaire, unidirectionel et le profil de vitesse est parabolique : il s'agit du profil laminaire de Hagen-Poiseuille, qui est une solution triviale des équations de NavierStokes [11]. La transition vers un écoulement turbulent

\footnotetext{
${ }^{a}$ Auteur pour correspondance : duguet@mech.kth.se
}

a été mise en évidence dès les travaux expérimentaux d'Osborne Reynolds [14]. Elle se manifeste, lorsque Re est inférieur à 2300, par l'existence de « bouchons turbulents », des structures turbulentes localisées qui se propagent avec une vitesse d'environ $0,9 U$, dont la longueur est de l'ordre de $20 D[23,24]$. Pour des plus grandes valeurs de $R e$, on constate l'apparition de « bouffées turbulentes » localisées (slugs) qui envahissent progressivement l'aval de la conduite [24]. Une valeur critique de $R e$ communément admise est 2000. Cependant, cette valeur critique est fortement dépendante des conditions expérimentales, et peut être repoussée au moins jusqu'à $10^{5}$ [10]. Une méthode désormais classique pour expliquer l'apparition de l'instationnarité dans un écoulement est de rechercher pour quels paramètres l'écoulement de base devient linéairement instable, puis d'étudier la dynamique non-linéaire des modes instables du système. Cette méthode s'est avérée d'une précision remarquable dans le cas des écoulements de Rayleigh-Bénard ou de TaylorCouette. Dans le cas de la conduite cylindrique, la théorie 
de l'instabilité linéaire n'est cependant d'aucune aide et ne permet pas de définir une valeur critique pour $R e$ [15]. En effet, il est généralement accepté que l'écoulement de Hagen-Poiseuille est linéairement stable quelle que soit la valeur de $R e$. La transition observée en pratique est donc une instabilité (convective) de l'écoulement à des perturbations d'amplitude finie. D'un point de vue théorique, les équations de Navier-Stokes peuvent être assimilées à un système dynamique de dimension infinie, dont l'état laminaire représente un point fixe stable [5]. La découverte récente de solutions tridimensionnelles d'amplitude finie a permis des progrès importants. Ces solutions des équations de Navier-Stokes sont des ondes progressives se propageant à une vitesse légèrement supérieure à $U[4$, $12,13,21]$. Certaines de ces ondes progressives peuvent être modulées périodiquement en temps via une bifurcation de Hopf [2]. Toutes ces solutions sont linéairement instables, et possèdent une longueur d'onde axiale de l'ordre de quelques diamètres. L'existence de ces solutions d'amplitude finie permet de comprendre la dynamique transitionelle des équations de Navier-Stokes lorsque des conditions aux limites périodiques sont imposées avec une période axiale $L \sim 5 D$. Il a en effet été remarqué que certaines de ces solutions, si elles sont perturbées de façon infinitésimale, évoluent soit vers l'état laminaire soit vers un état turbulent, en fonction du signe de la perturbation [18]. Cette propriété permet une meilleure compréhension de la topologie dans l'espace des phases de la frontière laminaire-turbulent $\Sigma$, le sous-espace invariant qui délimite les bassins d'attraction respectifs des états laminaire et turbulent. Un attracteur existe sur $\Sigma$, appelé « état limite». Il est bâti autour d'un réseau de solutions assimilées à des pointsselles ou à des orbites périodiques, et $\Sigma$ représente l'union de leurs variétés stables [3]. Cependant, cette condition de périodicité spatiale est quantitativement trop restrictive pour permettre l'existence des structures localisées observées expérimentalement. Cette étude présente la simulation numérique d'états limites pour une conduite périodique dont la longueur est d'un ordre de grandeur supérieure, ici $L=33,5 \mathrm{D}$. L'utilisation d'un modèle, dit $(2+\varepsilon)$ et dérivé de la discrétisation spectrale incomplète des équations de Navier-Stokes [22], permet de simplifier la dynamique de l'état limite, et ainsi de reconstruire un scénario potentiel pour décrire la transition et l'apparition des bouffées turbulentes du point de vue des systèmes dynamiques.

\subsection{Méthode numérique}

Le champ de vitesse tridimensionnel $\boldsymbol{u}=(u, v, w)$ et la pression $p$ associés aux perturbations à l'état laminaire sont régis par les équations de Navier-Stokes incompressibles, qui s'écrivent en variables adimensionnées :

$$
\begin{aligned}
\nabla \cdot \boldsymbol{u} & =0 \\
\frac{\partial \boldsymbol{u}}{\partial t}+(\boldsymbol{u} \cdot \nabla) \boldsymbol{u} & =-\nabla p+\frac{1}{R e} \Delta \boldsymbol{u},
\end{aligned}
$$

avec des conditions aux limites de non-glissement aux parois :

$$
\boldsymbol{u}(r=1)=\mathbf{0}
$$

L'algorithme, commun à la simulation numérique directe et au modèle $(2+\varepsilon)$, a déja été présenté dans une publication antérieure [22]. Les équations de Navier-Stokes, considérées dans un système de coordonnées cylindricopolaires $(r, \theta, z)$, sont reformulées via une décomposition poloïdale-toroïdale $\boldsymbol{u}=\boldsymbol{\nabla} \times(\Psi \hat{\boldsymbol{z}})+\boldsymbol{\nabla} \times \boldsymbol{\nabla} \times(\Phi \hat{\boldsymbol{z}})$. Chacun des potentiels scalaires $\Psi$ et $\Phi$ est discrétisé à l'aide d'un schéma de différences finies compactes dans la direction radiale, et d'une décomposition pseudo-spectrale sur des modes de Fourier dans les directions $\theta$ et $z$ (imposant de fait une condition de périodicité axiale). Par exemple, la décomposition de $\Phi$ à un noud radial $r_{j},(j=1, \ldots, N)$, s'écrit :

$$
\Phi\left(r_{j}, \theta, z, t ; \alpha, m_{0}\right)=\sum_{k=-K}^{K} \sum_{m=-M}^{M} \Phi_{j k m}(t) \mathrm{e}^{i\left(m_{0} m \theta+\alpha k z\right)}
$$

La symétrie rotationnelle $R_{m_{0}}:(u, v, w)(r, \theta, z) \stackrel{(}{\rightarrow}$ $(u, v, w)\left(r, \theta+\frac{2 \pi}{m_{0}}, z\right)$, avec $m_{0}$ entier, peut également être imposée. La résolution numérique est décrite par un quadruplet $\left(N, M, K, m_{0}\right)$, et le système dynamique correspondant possède ainsi $O(8 M N K)$ degrés de liberté. L'intégration en temps est assurée par un schéma de Crank-Nicholson pour les termes diffusifs et un schéma de type Euler prédicteur/correcteur pour les termes nonlinéaires [22]. La règle des $3 / 2$ est utilisée pour éviter les effets de dealiasing. La condition de débit constant est imposée via un terme de forçage homogène, réévalué à chaque pas de temps. Nous considérons dans cette étude deux cas différents :

- un cas $« 3 D » \operatorname{avec}\left(N, M, K, m_{0}\right)=(40,24,256,1)$;

- le cas du modèle « $(2+\varepsilon) » \operatorname{avec}\left(N, M, K, m_{0}\right)=$ $(35,1,256,3)$.

Le cas $3 D$ correspond à une très bonne discrétisation spatiale dans toutes les directions (en pratique 7 décades du spectre d'énergie sont représentées). La dimension du système dynamique associé est d'environ $2 \times 10^{6}$. Le modèle $(2+\varepsilon)$ conserve un nombre suffisant de modes dans les directions axiale et radiale, mais ne garde dans la direction azimuthale que les modes $-3,0,3$. Ces modes permettent en pratique de capturer les cycles non-linéaires de régénération de la vorticité axiale observés au sein d'un écoulement turbulent [20]. Ce modèle est numériquement stable, il a été utilisé pour reproduire avec succès les structures localisées du type bouchons ou bouffées turbulentes sans modfication quantitative notable des quantités importantes telles que le coefficient de frottement aux parois, moyennant un décalage de la valeur de $R e$. Son intérêt réside dans la simplification de la dynamique qui résulte de la suppression de certaines interactions nonlinéaires entre modes de Fourier azimutaux. 


\section{2 États limites}

\subsection{Trajectoires critiques}

Tout au cours de cette étude, deux quantités énergétiques sont considérées :

$$
\begin{aligned}
E_{3 D}(t) & =\int_{0}^{L} \int_{0}^{2 \pi} \int_{0}^{1}\left(\boldsymbol{u}-\langle\boldsymbol{u}\rangle_{z}\right)^{2} r \mathrm{~d} r \mathrm{~d} \theta \mathrm{d} z \\
E_{\mathrm{st}}(z, t) & =\int_{0}^{2 \pi} \int_{0}^{1}\left(w_{z}-\left\langle w_{z}\right\rangle_{\theta}\right)^{2} r \mathrm{~d} r \mathrm{~d} \theta
\end{aligned}
$$

où \langle\rangle$_{z}$ et \langle\rangle$_{\theta}$ correspondent respectivement à des moyennes spatiales selon la direction axiale et azimuthale. $E_{3 D}(t)$ représente l'énergie associée aux perturbations tridimensionnelles à l'écoulement moyen. $E_{\mathrm{st}}(z, t)$ mesure, à une position axiale donnée, l'énergie associée aux modulations azimuthales de la vitesse axiale. Des trajectoires évoluant sur la frontière laminaire-turbulent $\Sigma$ sont déterminées numériquement en utilisant un algorithme de bissection. L'amplitude d'une condition initiale donnée est ajustée par dichotomie jusqu'à la précision machine, de telle façon que la trajectoire associée n'évolue ni vers l'état laminaire $\left(E_{3 D}=0\right)$, ni vers l'état turbulent $\left(E_{3 D}\right.$ atteint des valeurs élevées) [3]. Le régime intermédiaire mis en évidence par cette bissection peut être suivi pendant un temps de l'ordre de $500 D / U$. De façon importante, le régime ainsi déterminé ne dépend (après une courte phase transitoire) plus de la condition initiale choisie, ce qui justifie son appellation d'état limite (ou encore « edge state $»[16])$.

Dans le cas $3 D$, l'état limite calculé pour $R e=$ 2000, 3000, 4500 et 6000 est chaotique en temps (comme dans le cas des courtes conduites). Il s'agit d'un régime où les perturbations à l'état laminaire sont localisées dans la direction axiale, indépendamment du caractère localisé ou non de la condition initiale. Lorsque $R e$ augmente (voir Fig. 1), l'état limite voit son énergie $E_{3 D}(t)$ décroître en moyenne. Il est également de plus en plus localisé dans la direction radiale (voir Fig. 2). Il est intéressant de constater que l'état critique reste localisé en $z$ indépendemment de la nature du régime turbulent associé (turbulence localisée pour $R e=2000$ ou délocalisée pour $R e \geq 3000)[8,22]$. Le cas $(2+\varepsilon)$ calculé pour $R e=10000$ montre que l'état limite converge asymptotiquement vers un état d'énergie stationnaire. Il s'agit d'une onde progressive localisée dans les directions axiale et radiale, se propageant avec une vitesse axiale $c=1,06 U$. Le cisaillement est prédominant près d'une couche critique située à $r=r_{\mathrm{c}}=0,67 \mathrm{D} / 2$ (cf. Fig. 3 ). Il est à noter que l'existence d'ondes progressives en conduite cylindrique a déjà été envisagée dans la limite inviscide, même si l'hypothèse d'une symétrie $m_{0}=3$ avait été rejetée [17]. Une couche critique d'épaisseur $O\left(R e^{-\frac{1}{3}}\right)$, résultant de la compétition locale entre effets inertiels et effets de diffusion a été prédite là où la vitesse du profil laminaire égale la célérité de l'onde en question (voir aussi le cas $3 \mathrm{D}$ [19] pour des ondes en conduites courtes). On notera ici que le profil laminaire en $2 U\left(1-r^{2}\right)$ atteint une valeur de $1,06 U$ pour $r=0,685 D / 2$, ce qui représente un
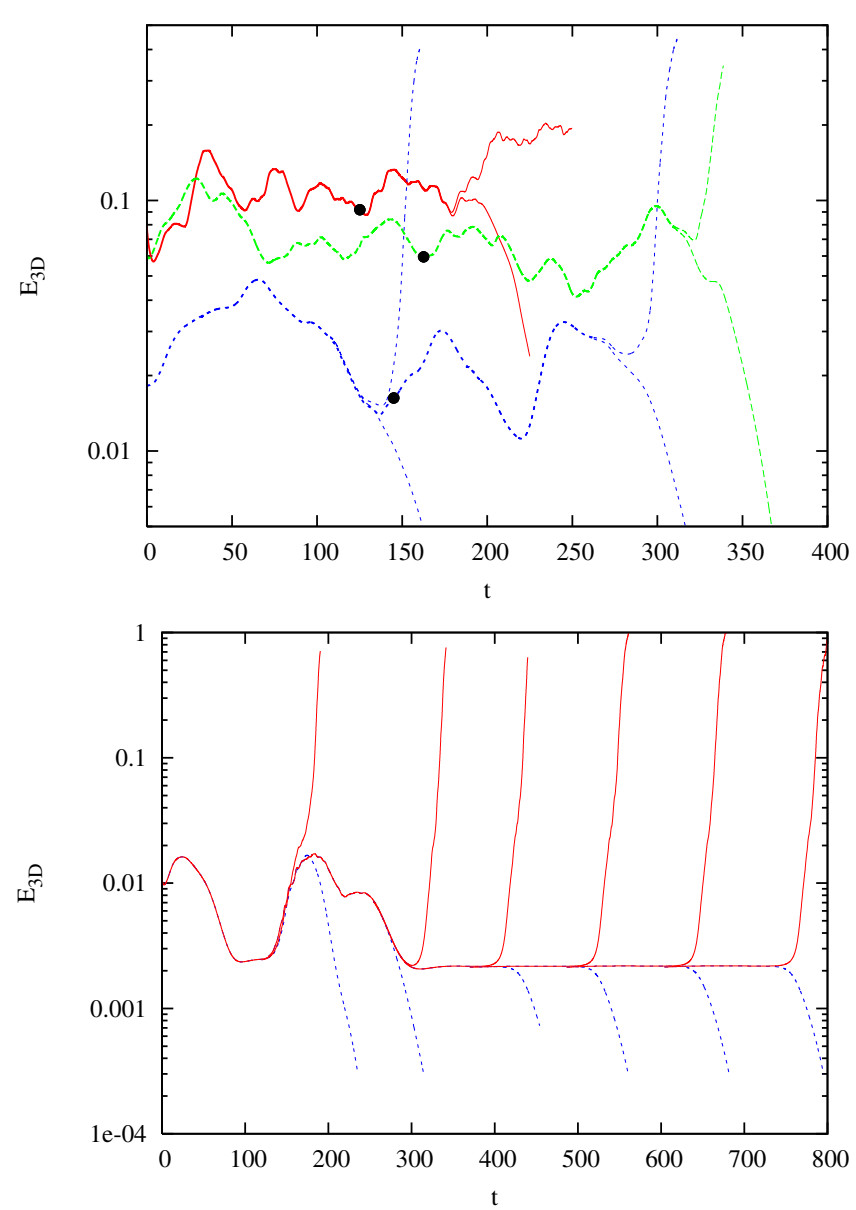

Fig. 1. $E_{3 D}(t)$ pendant le processus de bissection menant à des trajectoires critiques. En haut : cas $3 D$ pour $R e=2000$ (rouge), $R e=3000$ (vert) et $R e=6000$ (bleu). En bas : bissection pour le modèle $(2+\varepsilon)$ pour $R e=10000$.
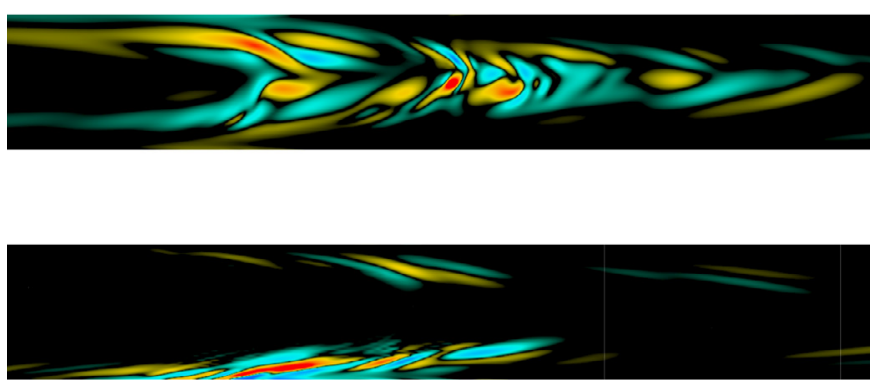

Fig. 2. Champ de vorticité axiale associé aux états limites dans une section $\theta=$ cste, pour $R e=3000$ (en haut) et $R e=$ 6000 (en bas). Seule la partie du domaine où l'écoulement est non-laminaire est représentée sur la figure.

excellent accord avec la théorie asymptotique vue la valeur modérée de $R e$. De plus, on observe sur la figure 3 (haut) un déphasage du champ de vitesse axiale de part et d'autre de la couche limite, un fait qui confirme également la prédiction asymptotique. 

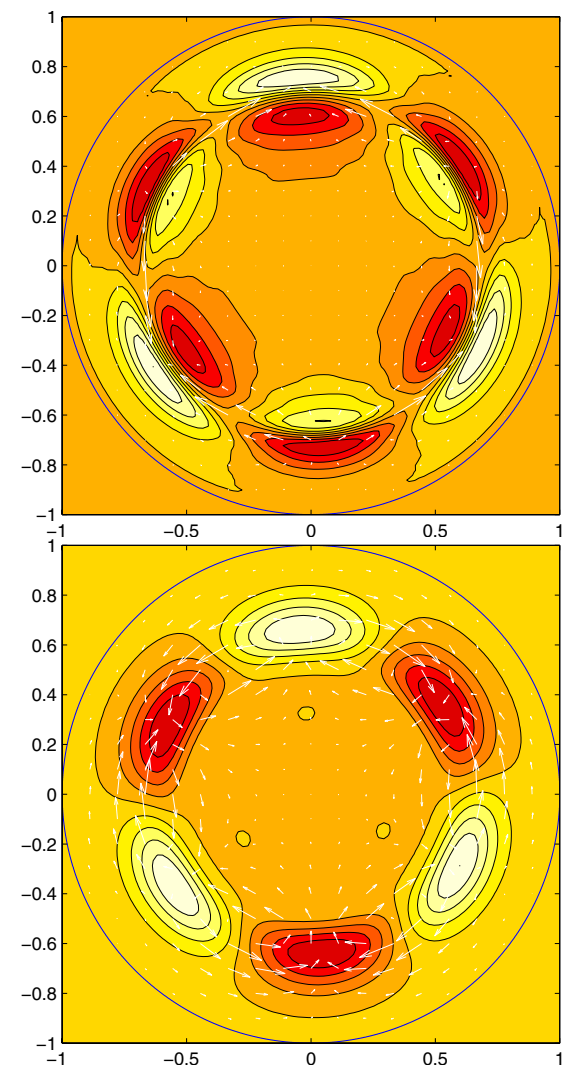

Fig. 3. Champ de vitesse associé à l'onde progressive du modèle $(2+\varepsilon)$ pour $R e=10000$. Coupe transversale près $\mathrm{du}$ front amont (gauche) et du front aval (droite).

\subsection{Trajectoires supercritiques}

Par construction, toute trajectoire partant d'une condition initiale dont l'énergie est légèrement supérieure à l'amplitude critique associée évolue d'abord de façon transitoire vers l'état limite, qui est instable, puis vers l'état turbulent. L'étude de telles trajectoires divergeant de l'état limite, dites supercritiques, est un bon point de départ pour la description des bouffées turbulentes, car elles correspondent à des conditions initiales reproductibles et d'énergie initiale minimale, par opposition à des réalisations expérimentales plus difficilement contrôlables. Une telle condition initiale correspond en principe à ce qu'observerait un expérimentateur qui augmenterait lentement l'amplitude initiale d'une perturbation au-delà du seuil critique, avec une précision infinie. Des trajectoires supercritiques ont été calculées dans le cas $3 D$ pour $R e=3000$, et dans le cas $2+\varepsilon$ pour $R e=10000$. L'énergie $E_{\mathrm{st}}(z, t)$ - associée à l'amplitude des modulations azimuthales de la vitesse axiale appelées stries est représentée dans les diagrammes espace-temps des figures 4 et 5. L'utilisation d'un repère en mouvement avec la vitesse de débit $U$ est utile à la compréhension du mécanisme responsable de l'expansion. Dans les deux cas $3 D$ et $(2+\varepsilon)$, il est notable que la région active de l'écoulement est délimitée par deux fronts. Dans le cas $3 D$, le système visite initialement le voisinage de l'état limite. La vitesse du front aval est peu modifiée pendant le processus, alors que la vitesse amont décélère brusquement vers $t=25$ d'une valeur supérieure à $U$ à une valeur inférieure à $U$. Dans le cas $3 D$, la décélération soudaine $\mathrm{du}$ front amont se produit après ue phase d'augmentation rapide des gradients axiaux de la quantité $E_{\mathrm{st}}(z, t)$ de part et d'autre de l'interface. La visualisation du champ de vorticité pour $t=25$ met en évidence, proche de l'interface amont, des structures tourbillonnaires ponctuelles du type Kelvin-Helmholtz (voir Fig. 6). Ces structures en « œil de chat» sont absentes de la structure de l'état limite. Ces structures tourbillonnaires se propagent en aval, se déstabilisent et induisent une activité turbulente dans la zone amont initalement laissée laminaire par le passage de l'onde initiale. Ceci suggère tout d'abord que le front amont associé à l'état limite est initialement sujet à une instabilité de cisaillement, directement liée à l'instabilité propre de l'état limite. Cette instabilité de cisaillement est ensuite responsable de la production turbulente à l'interface amont de la bouffée. Le nouveau front amont qui sépare la zone laminaire en amont de la nouvelle zone turbulente peut être de nouveau sujet à une instabilité de cisaillement. Vu d'un repère en mouvement à la vitesse de l'état limite, il s'agit d'un mécanisme d'instabilité autoentretenu qui permet à l'interface laminaire/turbulent de se propager vers l'amont. La propagation différentielle des deux fronts se manifeste par une expansion axiale de la bouffée turbulente, qui envahit progressivement tout le domaine de calcul. C'est ainsi la région amont de la structure localisée, par son instabilité de cisaillement, qui est le moteur du processus de contamination en amont de l'écoulement laminaire par l'écoulement turbulent à $R e=3000$. Le cas $2+\varepsilon$ ne présente pas de différence notable, si ce n'est une allure plus symétrique de la phase d'expansion spatiale dans le diagramme $(z-U t, t)$ (voir Fig. 5). Ceci suggère un rôle accru du front amont dans le processus d'expansion à haut $R e$. Dans le cas $3 D$, d'autres trajectoires supercritiques ont été calculées par la même méthode pour $R e=2000,4500$ et 6000 . Pour $R e \geq 4500$, la résolution numérique a été poussée à $(60,48,384,1)$. La vitesse de propagation des fronts amont et aval a été mesurée par interpolation à partir de diagrammes spatiotemporels analogues à celui de la figure 4. Ces vitesses de front, fonctions de la valeur de $R e$, sont comparées aux résultats expérimentaux les mieux documentés sur la figure 7. L'accord est très satisfaisant et la marge d'erreur est de l'ordre de la variabilité expérimentale. Ceci confirme que l'approche numérique retenue ici permet de simuler des bouffées turbulentes de façon réaliste.

\section{Discussion}

La trajectoire supercritique étudiée dans la section précédente peut être interprétée du point de vue de la théorie des systèmes dynamiques. L'interprétation est immédiate dans le cas du modèle $(2+\varepsilon)$, et permet une généralisation au cas $3 D$. Dans le cas du modèle $(2+\varepsilon)$, l'état limite est une onde progressive, c'est donc un attracteur relatif sur $\Sigma$. Cette onde progressive étant atteinte 


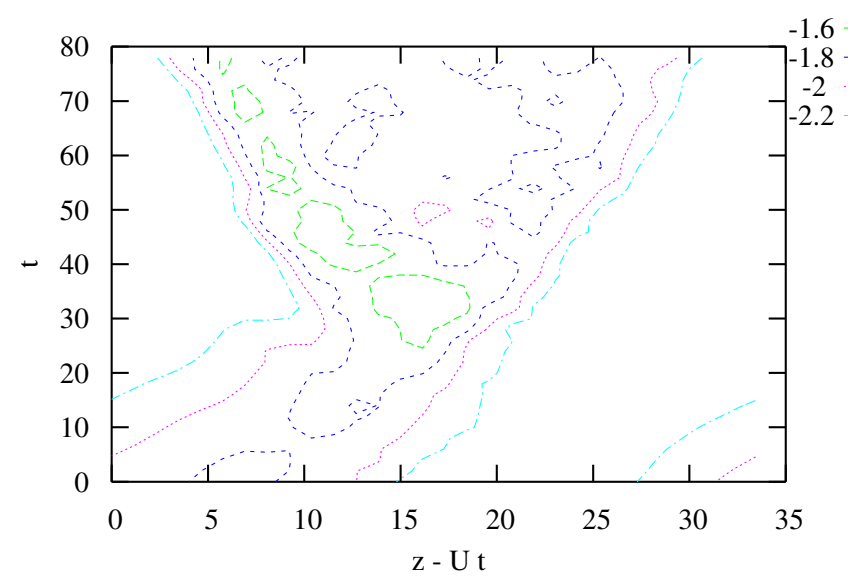

Fig. 4. $\log _{10}\left(E_{\mathrm{st}}(z-U t, t)\right)$ pour une trajectoire légèrement supercritique. Cas $3 D, R e=3000$.

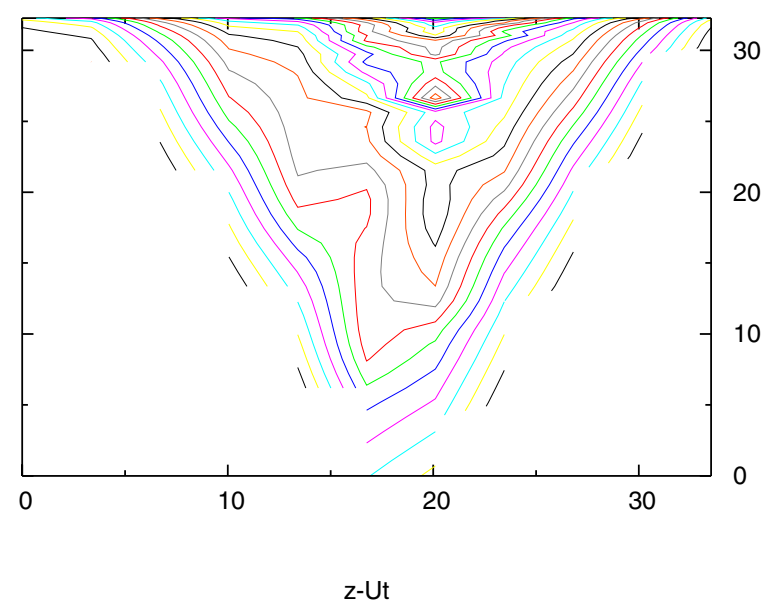

Fig. 5. $\log _{10}\left(E_{\mathrm{st}}(z-U t, t)\right)$ pour une trajectoire légèrement supercritique. Cas $(2+\varepsilon), R e=10000$.

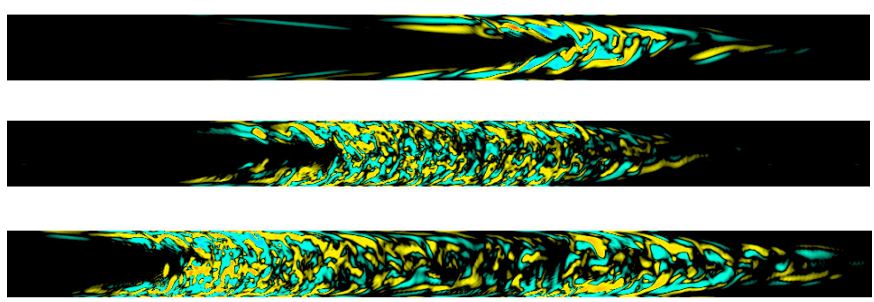

Fig. 6. Champ de vorticité axiale instantané dans une section $\theta=$ cste, associé au développement d'une bouffée turbulente à partir de l'état limite, pour $R e=3000$ (voir aussi Fig. 3). La coordonnée axiale est indicative et la coordonnée radiale est dilatée par souci de clareté. Les 3 images correspondent, de haut en bas, au temps $t=25,40$ et 55 .

asymptotiquement par un processus de bissection, elle est instable dans le complémentaire de $\Sigma$, et sa variété instable est de dimension totale 1 . Le développement de la bouffée turbulente, observé figure 5, correspond à l'unique trajectoire le long de la variété instable unidimensionnelle associée à l'onde progressive. Dans les conduites périodiques de faible longueur d'onde, il a été montré que la prise en compte de symétries additionnelles

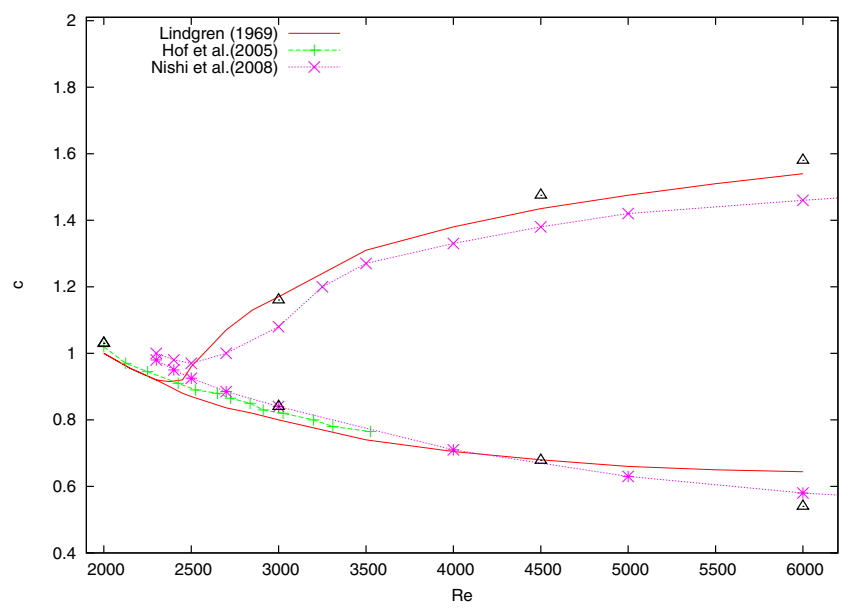

Fig. 7. Vitesses de propagation des fronts amont et aval en fonction de $R e$, normalisées par la vitesse de débit et obtenues par simulation numérique (triangles). Les résultats sont comparés aux estimations expérimentales de Lindgren (1969), Hof (2005) et Nishi (2008).

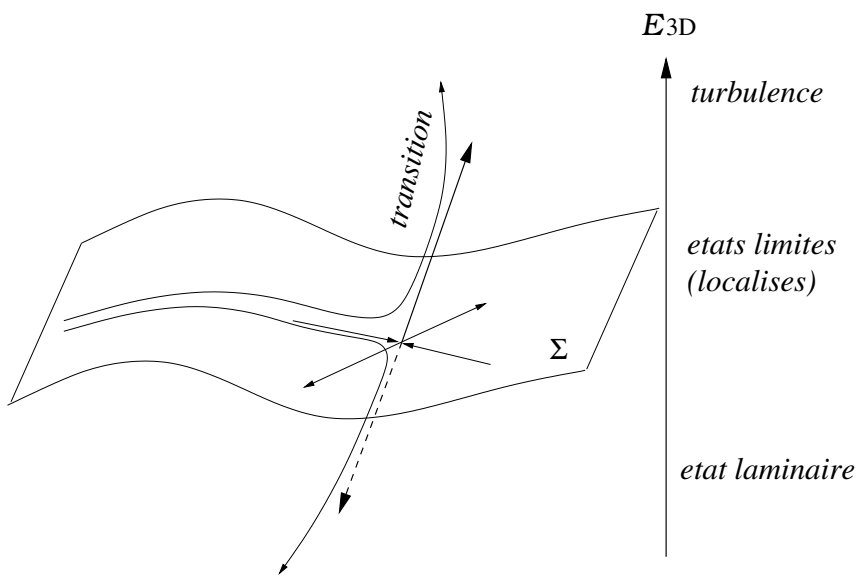

Fig. 8. Schéma de la topologie de l'espace des phases à proximité de la frontière laminaire-turbulent $\Sigma$.

est susceptible de réduire la dimension de la variété instable associée aux ondes progressives; par exemple un point-selle avec au moins deux dimensions instables peut devenir un attracteur relatif sur $\Sigma[3]$. De façon analogue, l'attracteur relatif du modèle $(2+\varepsilon)$ est l'analogue symétrique des ondes progressives du cas $3 D$. La variété instable associée à ces ondes $3 D$ a une dimension au moins égale à 2 puisqu'aucun attracteur relatif n'a été identifié. Ces ondes progressives correspondent également à des point-selles (ou à des orbites périodiques) sur $\Sigma$, expliquant ainsi la dynamique chaotique visible sur la figure 1 (gauche). Un schéma qualitatif de la dynamique proche du seuil est représenté sur la figure 6 . La notion de point-selle sur cette figure est simplificatrice, et correspond probablement en réalité à un enchevêtrement hétérocline entre différentes solutions. L'identification exacte et la continuation dans l'espace des paramètres de chacune de ces solutions localisées (dans le cas $3 D$ ) est laissée pour le futur. C'est une tâche numérique difficile qui nécessite des 
solveurs performants du type Newton-Krylov, capables de prendre en compte de très grands systèmes. Du point de vue physique, l'interprétation de la dynamique proche du seuil critique est la suivante : si l'énergie initiale associée à une perturbation n'est pas suffisante, le système peut subir une croissance transitoire, approcher momentanément une des ondes progressives sur $\Sigma$, puis relaminariser. Au delà du seuil critique, le système approche également cette onde progressive de façon transitoire, mais l'énergie s'accumule près du front amont et le cisaillement devient alors trop important. Le front amont devient instable et produit des fluctuations qui délimitent une nouvelle interface laminaire-turbulent. L'instabilité de cette nouvelle interface permet à la turbulence, par effet domino, d'envahir tout le domaine.

\section{Références}

[1] A.G. Darbyshire, T. Mullin, Transition to turbulence in constant-mass-flux pipe flow, J. Fluid Mech. 289 (1995) 83

[2] Y. Duguet, C. Pringle, R.R. Kerswell, Relative periodic orbits in transitional pipe flow, Phys. Fluids 20 (2008) 114102

[3] Y. Duguet, A.P. Willis, R.R. Kerswell, Transition in pipe flow: the saddle structure on the boundary of turbulence, J. Fluid Mech. 613 (2008) 255-274

[4] H. Faisst, B. Eckhardt, Travelling waves in pipe flow, Phys. Rev. Lett. 91 (2003) 224502

[5] R.R. Kerswell, Recent progress in understanding the transition to turbulence in a pipe, Nonlinearity 18 (2005) R17-R44

[6] B. Hof, C.W.H. van Doorne, J. Westerweel, F.T.M. Nieuwstadt, Turbulence regeneration in pipe flow at moderate Reynolds numbers, Phys. Rev. Lett. 95 (2005) 214502

[7] E.R. Lindgren, Propagation Velocity of Turbulent Slugs and Streaks in Transition Pipe Flow, Phys. Fluids 12 (1969) 418

[8] F. Mellibovsky, A. Meseguer, T.M. Schneider, B. Eckhardt, Transition in localised pipe flow turbulence, Phys. Rev. Lett. 103 (2009) 054502

[9] M. Nishi, B. Unsal, F. Durst, G. Biswas, Laminar-toturbulent transition of pipe flows through puffs and slugs, J. Fluid Mech. 614 (2008) 425-446
[10] W. Pfenniger, Transition in the inlet length of tubes at high Reynolds numbers, Boundary Layer and Flow Control, in: G.V. Lachman (éd.), 1961, Vol. 970

[11] J.L.M. Poiseuille, Recherches experimentales sur le mouvement des liquides dans les tubes de très petits diamètres, C.R. Acad. Sci. 11 (1840) 961

[12] C.C.T. Pringle, R.R. Kerswell, Asymmetric, helical and mirror-symmetric travelling waves in pipe flow, Phys. Rev. Lett. 99 (2007) 074502

[13] C.C.T. Pringle, Y. Duguet, R.R. Kerswell, Highlysymmetric travelling waves in pipe flow, Phil. Trans. Roy. Soc. A 367 (2009) 457-472

[14] O. Reynolds, An experimental investigation of the circumstances which determine whether the motion of water shall be direct or sinuous and of the law of resistance in parallel channels, Phil. Trans. Roy. Soc. 174 (1883) 935-982

[15] P. Schmid, D. Henningson, Stability and transition in shear flows, Springer, 2001

[16] T.M. Schneider, B. Eckhardt, J.A. Yorke, Turbulence transition and the edge of chaos in pipe flow, Phys. Rev. Lett. 99 (2007) 034502

[17] F.T. Smith, R.J. Bodonyi, Amplitude-dependent neutral modes in the Hagen-Poiseuille flow through a circular pipe, Proc. R. Soc. Lond. A 384 (1982) 463

[18] R.R. Kerswell, O.R. Tutty, Recurrence of Travelling Waves in Transitional Pipe Flow, J. Fluid Mech. 584 (2007) 69-102

[19] D. Viswanath, The critical layer in pipe flow at high Reynolds number, Phil. Trans. Royal Soc. 367 (2009) $561-576$

[20] F. Waleffe, On the self-sustaining process in shear flows, Phys. Fluids 9 (1997) 883-900

[21] H. Wedin, R.R. Kerswell, Exact coherent structures in pipe flow: travelling wave solutions, J. Fluid Mech. 508 (2004) 333-371

[22] A.P. Willis, R.R. Kerswell, Turbulent dynamics of pipe flow captured in a reduced model: puff relaminarisation and localised "edge" states, J. Fluid Mech. 619 (2009) 213-233

[23] A.P. Willis, J. Peixinho, R.R. Kerswell, T. Mullin, Experimental and theoretical progress in pipe flow transition, Phil. Trans. Roy. Soc. A 366 (2009) 2671-2684

[24] I.J. Wygnanski, F.H. Champagne, On transition in a pipe. Part 1. The origin of puffs and slugs and the flow in a turbulent slug, J. Fluid Mech. 59 (1973) 281-351 